\title{
LOS SISTEMAS DE APOYO ENTRE IGUALES (SAI) Y SU CONTRIBUCIÓN A LA CONVIVENCIA ESCOLAR
}

\author{
THE PEER SUPPORT SYSTEMS \\ AND ITS CONTRIBUTION TO CONVIVENCIA
}

\author{
José María Avilés Martínez \\ Universidad de Valladolid (España) \\ aviles@uva.es
}

\section{RESUMEN}

La convivencia escolar se considera hoy un ámbito preferente del trabajo preventivo en los contextos educativos para conseguir el equilibrio emocional y el bienestar personal y social del alumnado. También es un factor de actuación destacado para la educación en valores y la mejora del rendimiento integral del alumnado a largo plazo. Una de las herramientas más adecuadas que se utiliza en la convivencia escolar es proporcionar al alumnado presencia y voz, a través de diversas estructuras de participación y gestión. En los centros educativos funcionan diversos Sistemas de Apoyo entre Iguales (SAI): Acogida, Ayuda, Mediación, Tutoría o Mentoría. De forma intencional y diferenciada, cada uno de ellos da protagonismo al alumnado en la gestión de su propia convivencia. En este artículo, se analizan las diversas modalidades de apoyo entre iguales, sus características, y las distintas formas como se desarrollan hoy en las escuelas e institutos, destacando las potencialidades que cada uno de ellos posee. Por otra parte, se indican las habilidades que el alumnado desarrolla con su participación y las necesidades formativas y condiciones de implantación precisas para conseguir de ellos una efectividad manifiesta en los centros educativos y un encaje adecuado en otras estructuras escolares.

Palabras clave: Convivencia Escolar, Sistemas de Apoyo entre Iguales (SAI), Conflicto, Maltrato entre Iguales.

\begin{abstract}
Convivencia is now considered a preferred area of preventive work in educational contexts to achieve emotional balance and social well-being of students. It is also an intervention factor for the education in values and the improvement of the integral performance of the students in the long term. One of the most appropriate tools used in Convivencia is to provide students with presence and voice, through various structures of participation and management. In the schools there are several Support Systems (SAI): Partnering, Help Teams, Mediation, Tutoring or Mentoring. In an intentional and differentiated way, each one of them gives prominence to the students in the management of their own coexistence. In this paper, we analyze the different modalities of peer support, their characteristics, and the different ways
\end{abstract}


they are developed today in schools and high schools, highlighting their potentialities that each one of them has. On the other hand, they indicate the skills that the students develop with their participation and the training needs and precise implementation conditions to obtain from them in schools a manifest effectiveness and an appropriate fit in other structures of the educational context.

Keywords: Convivencia, Peer Support, Conflict, Bullying.

\section{INNOVACIÓN EDUCATIVA Y CONVIVENCIA ESCOLAR}

Muchos de los proyectos y metodologías que tratan de cambiar la escuela hoy usan la convivencia escolar como escenario para modificar las relaciones, los mensajes, las formas de hacer o, incluso, los contenidos de los procesos de enseñanza aprendizaje en el aula y en los itinerarios formativos del alumnado. Cada vez más se da carta de naturaleza a contenidos y procesos que tienen que ver con lo emocional, social, o moral para conseguir objetivos cognitivos, conductuales o de rendimiento en el alumnado.

El trabajo en convivencia supone establecer de forma compartida principios y líneas de actuación en la comunidad que permitan conseguir objetivos orientados a un fin, y que guíen la estimulación y trabajo en campos como el emocional, grupal, social o moral.

Estos principios orientarán la toma de decisiones compartida y responsable y las actuaciones planificadas e intencionales que se pongan en marcha, facilitando a cada persona el papel y la voz necesarias para entender y realizar el trabajo en convivencia como una tarea compartida y corresponsable.

Esta labor se ha de inspirar de algunos principios básicos:

- Ha de ser un trabajo intencional y planificado, con actuaciones programadas en el currículo escolar y en la vida de la escuela y de su comunidad educativa.

- Tiene que ser principalmente preventivo. Debe evitar enfoques paliativos o reactivos. Y se ha de entender, que o hay un trabajo proactivo o no hay trabajo. Ocuparse de la convivencia en positivo previene que surjan problemas después.

- Es imprescindible que sea una empresa colectiva y comunitaria, en la que se impliquen todos los miembros de la comunidad en una misma dirección. Lealtad educativa (Avilés, 2012) entre el profesorado, el alumnado y las familias.

- Hemos de participar haciendo protagonistas a todos y todas, en la medida de sus capacidades, con el deber que les obliga y desde el derecho que les asiste ser parte de la comunidad. No bastará con que el profesorado asuma su liderazgo, sino que las familias y el alumnado deben tener su protagonismo en la gestión de la convivencia.

- Han de ser procesos vivos, nacidos desde la necesidad, siempre de abajo a arriba. Construidos desde un punto de vista colaborativo, si queremos que sean reales y sentidos.

- Cada sector tiene una responsabilidad respecto de la convivencia de la que no puede soslayarse. Ejemplos son la cohesión grupal fomentada en el seno de los grupos por el 
propio alumnado, con la orientación y la guía del profesorado. O la construcción de referentes que las propias familias han de cimentar con sus hijos e hijas en terrenos como el moral, en la misma dirección que el profesorado. O la cultura del cuidado del otro, que debe instalarse en cualquiera de los sectores, especialmente con aquellas personas más vulnerables.

- La visión holística del trabajo en convivencia permitirá que los árboles dejen ver el bosque y no se difuminen los objetivos finales que dan soporte socioemocional y moral al alumnado para construir de forma autónoma sus relaciones con el mundo, y en especial, con los iguales con los que conviven en un marco de ciudadanía democrática.

Hay estudios que relacionan clima escolar y bienestar emocional del alumnado, y más a largo plazo, con rendimiento académico (Cowie y Oztug, 2008; Hung, Luebbe, y Flaspohler, 2014; Lester y Cross, 2015; Pellegrini y Bartini, 2000; Zullig, Huebner y Patton, 2011).

En el clima escolar influyen muchas variables, algunas relacionadas con problemas de convivencia en el aula y su gestión por parte del profesorado. Variables como el estilo docente (Fernández, 2007), expectativas satisfechas del alumnado, motivación, reconocimiento personal, respuesta a los intereses y necesidades, gestión de los límites, supervisión y evaluación de normas (Vaello, 2003), entre otras, son factores de protección o riesgo que están detrás de problemas de convivencia como la disrupción, la indisciplina o la desmotivación. Otras variables como el equilibrio en la gestión del poder en el seno de los grupos (Rigby, 2002), su grado de cohesión interna, el aislamiento o rechazo de alguno de sus miembros (Cerezo, 2001), la participación de los iguales en el cuidado de los otros (Avilés, 2013; Avilés, Torres y Vián, 2008; Cowie y Jennifer, 2007; De Vicente y Fernández, 2014), son a su vez factores de protección y riesgo grupales, entre otros, para que surjan espontáneamente o no procesos de violencia interpersonal o bullying. De ahí la importancia de trabajar intencionalmente la convivencia en positivo en el seno de los grupos, para proporcionarles herramientas de defensa y prevención tanto del itinerario perturbador de la convivencia, disrupción, indisciplina o desmotivación, como del itinerario de la violencia en sí, agresión, violencia o maltrato (Avilés, 2006).

El papel que pueden desempañar los iguales en cualquiera de los sistemas de apoyo en que se pueden organizar, supone un respaldo fundamental al trabajo que pueda estar haciéndose desde otros sectores de la comunidad para la mejora de su bienestar emocional y social (Cowie y Smith, 2010), promoviendo una cultura del cuidado y autocuidado, y el sentido de protección para quien lo necesita, al trabajar el valor de observar las necesidades y estar pendientes de otras personas.

El clima escolar incide también en la efectividad del trabajo y el rendimiento escolar en el aula. Trabajar la convivencia y sus problemas los mejora y ayuda a alcanzar un mejor resultado. Evitar y prevenir situaciones de disrupción facilita la marcha de la clase y el ritmo de los procesos de enseñanza aprendizaje entre el alumnado y con el profesorado. Responder a los intereses de cada uno de los alumnos y alumnas adecuadamente favorece su motivación e incentiva sus iniciativas personales, ampliando la inclusión en el conjunto de la clase, al sentirse todos y todas en proceso de realización. Fuera del grupo, la ausencia de conflictos relevantes, aumenta las posibilidades de progreso, y dentro de él, no estar pendiente de problemas, amenazas o necesidad de defensa, permite 
a cada persona centrarse en lo que importa, avanzando en los procesos de aprendizaje y teniendo mayor estabilidad emocional y bienestar personal.

\section{Los Sistemas de Apoyo entre Iguales (SAI) en el marco de los Planes de Convivencia}

Un Sistema de Apoyo entre Iguales, en adelante SAI, es una estructura organizada en red en la escuela, supervisada por las personas adultas de la comunidad educativa, en la que participa de forma autónoma y protagonista el alumnado con el fin de proveer una asistencia de valor a sus iguales, que puede ser de diversa índole y complejidad, según la edad, la intención y/o la necesidad del grupo de convivencia. El apoyo que prestan estos Sistemas va desde el acompañamiento y la acogida en los más sencillos, a la mentoría y el consejo en los más complejos y avanzados (Cowie y Sharp, 1996), pasando por la amistad, la ayuda, o la mediación en los intermedios.

Lógicamente, cualquier SAI descansa educativamente en las decisiones tomadas en la comunidad, en su Plan de Convivencia. Marco en el que se va a inspirar y al que responderá desde sus características, según el Sistema que sea, y a unos principios comunes transversales a cualquiera de ellos.

Los SAI participan de unos principios que los asemejan:

- El alumnado es protagonista de la gestión de su convivencia. Tiene una autonomía de actuación que le confiere una responsabilidad proporcional a la edad y a la necesidad.

- Son Sistemas supervisados. A pesar de su autonomía, el alumnado, o a demanda o de oficio, mantiene contactos de supervisión con las personas adultas de la comunidad, que les orientan y ayudan en sus tareas, dudas y cuestionamientos.

- Son Sistemas sostenibles, en la medida de lo posible. Se busca que sea el propio personal de la comunidad educativa quien los mantenga, forme y renueve año a año, evitando cualquier dependencia externa que los condicione, aunque siempre es posible recurrir puntualmente a ellas para resolver necesidades concretas.

- Son Sistemas Visibles y Reconocidos en la comunidad. Son estructuras con respaldo institucional para actuar y visibilidad cotidiana en la vida del centro, incluso con distintivos reconocibles que los acreditan e identifican entre sus iguales.

- Se busca un liderazgo compartido. Para evitar individualismos y protagonismos indeseados, se promueve un funcionamiento en equipo para la toma de decisiones. Pensamos más en equipos que en individuos (Avilés, 2012, 2013; Avilés y Alonso, 2017a, 2017b; Avilés, Torres y Vián, 2008).

- Su fin es acompañar el desarrollo socioemocional y moral de sus iguales (Tognetta, Avilés, Rosario y Alonso, 2015). Por eso, son criterios importantes y preferentes para la selección de los y las candidatas, los que poseen perfiles de liderazgo positivo, desarrollo moral avanzado, ascendencia grupal, autoridad moral, ejemplo conductual o compromiso y coherencia personales (Naylor y Cowie, 1999, 2000). Además, como colaboran con sus pares en la mejora de las habilidades sociales y emocionales y en su desarrollo moral, de ahí la supervisión de todos los Sistemas. 
Por tratarse de Sistemas que forman parte del Plan de Convivencia de los centros, a su vez participan conjuntamente con otras estructuras y redes en algunas de estas tareas. Su propia existencia y funcionamiento depende de la actuación de otras estructuras que forma el profesorado para desarrollar sus planes de convivencia: Equipo de Convivencia o Tutoría de Convivencia. Un Equipo de Convivencia suele estar formado por profesorado y otros profesionales que trabajan la convivencia en el centro y que tienen a su cargo precisamente la implementación y supervisión de estos Sistemas, entre otras funciones. En muchos casos, son los miembros de estos equipos quienes terminan conformando figuras como la de Los Tutores y Tutoras de Convivencia (Avilés, García y Matéu, 2012), profesorado que además de sus tareas académicas relacionadas con un área o materia, asume dentro del centro esta otra función.

Tanto en las estructuras que conforman las personas adultas como en las que conforman los distintos SAI, se apuesta por el formato de equipo (Avilés, Torres y Vián, 2008) superando el más individualizado y personal (Fernández, Villoslada y Funes, 2002). Esto evita tanto los riesgos ya conocidos de un modelo individualista (excesivo protagonismo, actuaciones inadecuadas), como el peso, también conocido, que supone el rol individual para los sujetos (excesiva responsabilidad, indecisión en la toma de decisiones, negativa a participar).

Un modelo basado en el formato equipo (Avilés y Alonso, 2017a, 2017b), favorece que el alumnado se sienta más respaldado y acompañado por sus iguales; se acostumbra a compartir las decisiones tomadas en conjunto; evita el riesgo de actuación inadecuada por parte de alguno de sus miembros; interioriza el hábito de consensuar con el resto lo que es más favorable hacer en las situaciones y casos importantes; disminuye el sentido de responsabilidad individual en las actuaciones a acometer; se evitan protagonismos innecesarios; se aumenta la sensación del poder del apoyo en el alumnado ayudado; se potencia más el respeto y la confianza del grupo de iguales hacia los miembros del equipo; incluso, se facilitan los relevos de sus componentes durante el curso o en cada nuevo curso escolar.

Entendemos, pues, que es más enriquecedor actuar en equipo que hacerlo individualmente, por eso es bueno facilitar el apoyo a través del bloque. Porque lo que cada alumno o alumna aporta es fundamental para que funcione el conjunto, es necesario que cada uno se implique en ese trabajo.

\section{TIPOLOGÍA DE SISTEMAS DE APOYO ENTRE IGUALES (SAI)}

Los SAI provienen de la tradición educativa canadiense y estadounidense en los años setenta en el siglo pasado (Naylor, 2010). Aparecen en las Islas Británicas primero, y de ahí posteriormente por toda Europa. En España se incorporan apenas en los años noventa y en relación con iniciativas de resolución de conflictos y para la mediación. Posteriormente, se desarrollan y presentan también como apoyo a las situaciones de prevención del maltrato entre iguales, teniendo una extensión hoy en nuestros centros bastante amplia, aunque no generalizada.

Los SAI (Avilés, 2006, 2012, 2013; 2017a; 2017b; Avilés, Torres y Vián, 2008; Cowie y Fernández, 2006; Naylor y Cowie, 2000; Naylor, 2010) son trasladados al escenario preventivo de los problemas de convivencia escolar buscando los efectos facilitadores y las ventajas, que en 
otros campos como el del aprendizaje, proporcionaba el trabajo entre iguales (Gartner, Kohler y Riessman, 1971; Slavin, 1995).

Podemos distinguir modalidades diversas que responden a necesidades específicas.

\section{Acogida}

La modalidad de acompañamiento y acogida se ha ubicado dentro de las denominadas peer partnering (Sullivan, 2001) que tienen como tarea básica acoger, dar la bienvenida, amistad, compartir tiempo, dar apoyo y proporcionar orientaciones iniciales a quienes lo demandan o lo necesitan. Algunas de las formas que ha adoptado en nuestro entorno se han denominado Alumnado acompañante (De Vicente y Fernández, 2014), Amigos -Befrienders, (Sullivan, 2001) Apadrinamientos - Buddies (Fernández, Villoslada y Funes, 2002), Equipos de Acogida (Avilés, 2018, en prensa), entre otros.

Se trata de un Sistema que presta una tarea, sencilla pero básica, para la mejora de la convivencia en positivo en los grupos, y que puede servir para resolver uno de los problemas más frecuentes para algunos estudiantes en nuestros centros educativos, la integración del alumnado en sus grupos de referencia y convivencia. Problemas como la soledad, el aislamiento, la falta de amigos, el desconocimiento de compañeros en la clase, la participación en actividades grupales, etc., pueden minimizarse con el fomento y la aplicación en la convivencia de valores como el acogimiento, la compañía, el conocimiento mutuo o la amistad.

\section{Ayuda}

Denominados peer support (Cowie y Sharp, 1996), en los Sistemas de Ayuda se solicita de los que participan en ellos que sean capaces de proporcionar escucha, y ayuden a quienes tienen problemas, para que aprendan a buscarles salidas. Son sistemas que exigen, por tanto, la adquisición de un repertorio de habilidades previo a la participación del alumnado, que en algunos casos proporciona el propio profesorado del centro educativo a través de momentos, espacios formativos (Avilés, 2014), incluso materiales (Avilés y Alonso, 2017a, 2017b). La ayuda que se suele prestar se dirige a resolver problemas tan comunes como los casos incipientes de bullying o cyberbullying, la timidez, el aislamiento, la falta de aceptación dentro del grupo, las rencillas y envidias entre iguales,... La forma que se ha desarrollado de forma más generalizada es la de alumno ayudante o alumno ayuda (Fernández, Villoslada y Funes, 2002). Otras han funcionado a través de un equipo, como en el caso de los Equipos de Ayuda (Avilés, Torres y Vián, 2008), donde además de la formación para la prestación de la ayuda, se les da formación para tomar decisiones en equipo, y se prima la fuerza del equipo antes que la individual para ejercer y sentir la ayuda.

\section{Mediación}

Quizás sea este SAI el que ha tenido un desarrollo mayor en cuanto a la extensión de su generalización en los centros educativos de nuestro país (Zaitegi, et al., 2010). Su inclusión legislativa en leyes de carácter estatal (LOE, 2006), así como en normas de rango autonómico, no hicieron más que recoger prácticas que estaban dándose en los centros, como respuesta a la resolución pacífica y en positivo de los conflictos. Herederos de estudios que proclamaban los beneficios de este sistema (Close y Lechman, 1997; Cohen, Compton y Diekmann, 2000; Jones, 1998; Van Slyck y Stern, 1999), la implantación de la mediación en los centros ha perseguido la resolución 
de los conflictos de forma pacífica y el afrontamiento de la violencia en un escenario, el de los iguales, donde realmente se construyen los significados compartidos y legitimados de las conductas aceptables y no aceptables (Zaitegi et al., 2010). A diferencia de otros sistemas de apoyo, este aporta una función cívica, registrada públicamente en el marco de convivencia, donde los sujetos pueden llegar a alcanzar consensos y acuerdos, y estos llegar a tener rango de solución reconocida. Esto no siempre fue así. Al principio, las comunidades educativas de los centros comprobaron que el alumnado ensayaba poco a poco competencias con un fuerte valor educativo y transmitía ilusión a quienes participaban en el proyecto. Los centros comenzaron a observar las contradicciones en sus normas de organización y funcionamiento, las adaptaron a los nuevos sistemas y comprobaron cómo cambiaban las dinámicas, mejoraban las relaciones y se establecían vínculos más positivos. Se empezó a hablar y a desarrollar, entonces, una “cultura de la mediación”, que tomó cuerpo y función en la gestión de la convivencia (Boqué, 2005). Por tanto, es lógico ese reconocimiento y encaje en las estructuras del centro. Por eso, la mediación supera la propia gestión de las situaciones de conflicto y persigue sembrar cultura de mediación en la convivencia de quienes la practican. Entre personas y en las estructuras del centro y su sistema de relaciones (García Barreiro y Avilés, 2018 en prensa).

Las personas mediadoras intervienen en situaciones como conflictos entre iguales, malos entendidos, rencillas, disputas, confrontación de intereses, choque de necesidades, rivalidades,... etc.

La mediación también se puede aplicar entre desiguales, escolarmente hablando, cuando la cultura de mediación es práctica más extendida e interiorizada en la comunidad educativa. De esta otra forma, la mediación sirve para resolver conflictos entre el profesorado y el alumnado o el profesorado y las familias o el alumnado y las familias.

\section{Tutoría}

Como Sistemas de Apoyo entre Iguales, se han establecido diferencias entre Tutoría y Mentoría (Megginson y Clutterbuck, 2009), incluso con el coaching, como se puede apreciar en la Figura 1. Las tres formas quedan delimitadas en los espacios de lo que se llama mentoría (Sullivan, 2001), sin embargo, cada una tiene un lugar y características propias. Las tres pueden establecer una relación individual (el coaching, incluso grupal) entre quien presta el apoyo y quien lo recibe.

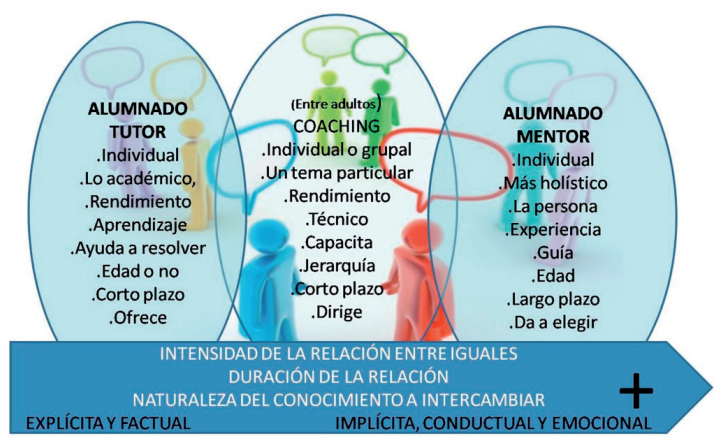

Figura 1: Diferencias entre Tutoring, Coaching y Mentoring (Megginson y Clutterbuck, 2009). Fuente: elaboración propia 
Los sistemas tutoriales se denominan peer tutoring (Topping, 1996) y se centran más en lo académico y la superación del rendimiento. Abordan aspectos más técnicos y a corto plazo. En esencia, consisten en emplear las destrezas académicas y personales de algunos alumnos o alumnas en alguna faceta o materia, para ponerlos al servicio de otros que los necesitan, estableciendo relaciones relativamente estables entre iguales para prestar ese apoyo de tutoría, supervisión o seguimiento en diversas actividades.

La prestación que ofrece un tutor o tutora está relacionada con la capacitación que tiene y proporciona al igual tutorado. Las formas más frecuentes de este Sistema son el Club de Deberes (De Vicente y Fernández, 2014) en que alumnado de cursos superiores ayuda en tareas escolares a alumnado que presenta dificultades; los Tutores Escolares (Durán y Vidal, 2004) que se orienta a la explicación de conceptos, mantenimiento de hábitos de trabajo y ejecución de tareas; o los Bancos de Tiempo, que el alumnado emplea para prestar tutoría en determinadas materias a otros que lo necesitan (De Vicente y Fernández, 2014).

\section{Mentoría}

El concepto de mentoría aplicado al marco escolar, refiere tareas de guía, supervisión, modelaje y facilitación hacia logros individuales, generalmente académicos, aunque no necesariamente curriculares, por ejemplo en la educación superior, pero también personales y relacionados con valores, de desarrollo moral, de obtención de metas y/o de superación personal y social en niveles escolares inferiores. Así, las tareas habituales de los mentores van dirigidas a mejoras de conducta, mejoras de absentismo en algunos casos, apoyo a iniciativas en el estudio, mejora del potencial de talento del alumnado que no encuentra en la escuela respuesta a sus intereses, afrontamiento de situaciones como el bullying o el cyberbullying, refuerzo de la autoestima y la autoconfianza para progresar, intentar minimizar el impacto de algunos cambios personales o familiares, mejorar de la experiencia y el desarrollo educativos, etc.

El Sistema de Mentoría se enfoca más a contenidos personales, de forma más holística y dirigidos a la superación personal y al logro de metas individuales. Los mentores establecen una relación más a largo plazo y más personal con quienes guían.

En la prevención del cyberbullying y los riesgos del mal uso de las redes sociales han surgido modelos de mentoría entre iguales como la figura de los cibermentores (Avilés, 2013, 2018, en prensa; Avilés y García Barreiro, 2016) que guían a sus compañeros en problemas relacionados con la privacidad, la comunicación y la gestión de las relaciones en las redes sociales.

En algunas escuelas la mentoría también la ha ejercido el adulto hacia los estudiantes, aproximándose más al modelo de coaching, adoptando sistemas como el de Tutoría de convivencia (Avilés, 2014; Avilés, García y Mateu, 2012), quienes gestionan los acuerdos reeducativos y seguimientos tutoriales de alumnado desfavorecido o con riesgo de fracaso escolar y personal. 


\section{LOS SAI COMO RESPUESTA A LOS PROBLEMAS DE CONVIVENCIA}

Como se acaba de indicar, cada SAI posee diferentes posibilidades de aplicación para la mejora de la convivencia, así como son distintas las habilidades que tiene que desplegar el alumnado para cumplir su tarea. Corresponde a los centros entender qué herramienta aplicar a cada situación y problemática, teniendo muy presente que no todos los SAI valen para resolver cualquier problema de convivencia. En este sentido, será muy acertada una doble diferenciación, por una parte un diagnóstico certero de la naturaleza de los problemas que queremos acometer, y por otra, la idoneidad de los SAI para resolver esas situaciones.

Es cierto que en la escuela los problemas se manifiestan de forma simultánea e interactuando mutuamente. Problemas de clima de aula, indisciplina o disrupción potenciados por situaciones de desmotivación y falta de interés; e incluso, insertos en situaciones de otra naturaleza como son los ambientes donde la violencia o el abuso se solapan. En este sentido, los profesionales de la educación deben tener presentes algunas separaciones que comparan constructos de distinto origen y que algunos autores (Avilés, 2015, Rigby, 2002) han remarcado de cara a la intervención. Es el caso, por ejemplo, de la necesaria distinción entre Conflicto y Maltrato, que atraviesa muchas situaciones de quiebra de la convivencia, pero que separa la naturaleza de los fenómenos y condiciona su forma de intervención. Y por ende, también el SAI más adecuado que emplear, para hacer tanto prevención como intervención, lo que condicionará a su vez también su efectividad. Esta vieja distinción (Rigby, 2002) enfrenta procesos en que el desequilibrio de poder es manifiesto (maltrato), con otros en los que el equilibrio de fuerzas permite a las partes confrontar, manifestar o defender sus posturas. Así vemos, que Sistemas como la ayuda o la mentoría se postulan como más idóneos para afrontar la prevención y resolución del maltrato, mientras otros como la mediación o la tutoría no lo son, quedando más para situaciones de conflicto o para apoyos académicos. A pesar de ello, todavía hoy se siguen presentando algunas propuestas que emplean sistemas tutoriales de forma generalizada para resolver problemas que no tienen, ni mucho menos, ese cariz ni condición; o se plantean espacios de mediación para hacer encontrarse a víctima y agresor en soluciones al acoso. Todo ello, propiciado por una cierta mercantilización, al considerar algunos temas como el del bullying. No obstante, se ha de decir que hemos de movernos en terrenos de grises, pero sabiendo en todo momento la naturaleza del fenómeno que se gestiona, para no emplear las estrategias equivocadas o no agravar situaciones, de por sí ya graves.

\section{ALGUNAS CONDICIONES DE IMPLANTACIÓN DE LOS SAI EN EL CONTEXTO ESCOLAR}

Evidentemente no hay una única manera de llevar a la práctica la implantación de un SAI en un contexto educativo. Se pueden desencadenar diversas acciones en una secuencia adaptada a la comunidad, que sirvan para los objetivos de puesta en marcha. Sin embargo, se indican algunos momentos y estructuras recomendables.

- Debe desarrollarse alguna acción de sensibilización en cada sector de la comunidad educativa que justifique y legitime el desarrollo del SAI. Se deben dar los pasos para que los miembros de la comunidad educativa se sientan motivados a apoyar la idea. 
- Se deben encajar esas figuras en las estructuras y planes del centro. El Proyecto Educativo, Plan de Convivencia, El Plan de Acción Tutorial, Las Tutorías. Así mismo, se debe hacer responsables a las estructuras del centro, si las hay, de la creación, funcionamiento y mantenimiento de los SAI. En este sentido sería recomendable contar con un Grupo de Convivencia, Responsable de Convivencia o Tutoría de Convivencia.

- Es conveniente marcar perfiles diferenciados en cada uno de los SAI que se pongan en marcha en los centros y destinarlos a cubrir necesidades o problemáticas diferentes, o al menos, complementarias. Se deben evitar duplicidades y solapamientos. Cada SAI debe responder a lo que se ha pensado para él.

- Se han de preparar a los grupos aula, si es que son ellos quienes deben hacer la elección de los componentes de los SAI, o establecer un mecanismo de selección de candidatos. En el primer caso, la preparación del grupo que elige a los componentes es crucial y supone gran parte del éxito o fracaso futuro del Servicio. En el segundo caso, quienes seleccionan al alumnado que componga el SAI, deben conocerlo bien y tener claros los criterios de perfil y habilidad que requieren los puestos.

- El alumnado que compone los SAI, aunque presente unas habilidades propias, necesita tener momentos de formación donde se le cultiven y contrasten esas destrezas explícitamente. Estos períodos, intensivos o puntuales, pueden ofrecerse después de la selección a los candidatos, y es bueno que sean asumidos por las personas adultas, que supervisarán y mantendrán el Servicio.

- Las personas responsables que componen las estructuras que sustentan los SAI (Grupo de Convivencia, Coordinación de Convivencia, Tutoría de Convivencia) es necesario que planteen la sostenibilidad del Servicio, combinando experiencia y novedad en la renovación de un SAI, o ideando, por ejemplo, itinerarios formativos y de paso para el alumnado, de los SAI más sencillos y generalizables, a los más complejos y personalizados, para facilitar el tránsito de las personas por los distintos SAI. Así, un centro que potencie la sostenibilidad de sus SAI, utilizará a alumnado que comienza en Equipos de Ayuda inicialmente, demostrando habilidad y buen hacer, para después pasando el tiempo, poder seleccionarlos y emplearlos, por ejemplo, como cibermentores cuando estudien en cursos superiores, formándolos para esa nueva tarea. Así, se aprovechará y se hará más sostenible el sistema, enriqueciendo las habilidades del alumnado y ofreciendo a la convivencia más recursos que poder utilizar.

\section{PROPUESTA DE UN ITINERARIO FORMATIVO PARA CADA SAI}

Sin duda, el itinerario formativo del alumnado que participa en uno o en varios SAI a lo largo de su escolarización es diverso y dependerá de las condiciones del centro educativo y las necesidades que el Servicio pretenda atender.

Se pueden indicar algunas habilidades comunes, que son necesarias emplear en cualquiera de ellos, en distinto grado y nivel, como son las de capacidad de presentación, comunicación o las de análisis de situaciones problemáticas. En Servicios de Apoyo como el de Ayuda y el de Mentoría 
es necesario formar al alumnado en la escucha activa, la gestión de emociones, y en situaciones de empatía y asertividad (Avilés y Alonso, 2017b), contextos que tienen que manejar en realidades como las del bullying y otras. En Servicios como el de Cibermentoría se hace necesario incorporar bloques formativos que incorporen habilidades técnicas como las informáticas o de creación audiovisual (Avilés y García Barreiro, 2016), aunque siempre se debe hacer primar en la selección de este alumnado su perfil sociomoral. En Servicios como el de Mediación, en el que básicamente se manejan conflictos, las habilidades de pensamiento y el ejercicio de la neutralidad son contenidos centrales de la formación. Cuando el formato elegido para constituir el servicio es el de equipo, como en los casos de Equipos de Acogida, Equipos de Ayuda, Equipos de Mediación o de Cibermentores, es imprescindible incorporar módulos formativos de trabajo en equipo y toma de decisiones por consenso.

En cualquier caso, se entiende que cada centro educativo conformará la formación, elaborará sus propios materiales o usará algunos disponibles (Alonso y Avilés, 2017a, 2017b; Avilés y Alonso, 2017a, 2017b; Boqué, 2005; Luengo, 2014; Torrego, 2001), y la impartirá al alumnado en diferentes momentos del curso, para hacer el procedimiento sostenible en el tiempo. En este sentido, el horario de la formación es un aspecto importante. No se trata de una formación al margen de los contenidos curriculares y del horario escolar. Cuanto más inserta esté en esos tiempos, más integrados y normalizados estarán los SAI en el Proyecto de Convivencia del centro.

Estas propuestas formativas pueden adoptar diversas formas y momentos. Conviene que haya momentos intensivos, pero otra formación puede ser puntual y a demanda. Por ejemplo, con la renovación anual de los SAI conviene realizar un curso de formación con el fin de aportar herramientas de trabajo al alumnado que componga el Servicio. En el transcurso de la práctica del SAI surgen nuevas necesidades, que pueden ser atendidas puntualmente por el profesorado del centro o personas externas a él, mediante sesiones que se pueden celebrar a lo largo del curso. Otras fórmulas pueden darse dentro del horario lectivo en algunas materias, pensadas con este fin o no. Incluso, algunas actividades pueden utilizar períodos no lectivos esporádicamente, por ejemplo, como parte de una jornada intensa de formación o un fin de semana conviviendo,...

Por ello, la secuencia formativa de los SAI es un tema central, que condiciona su preparación para la tarea efectiva que se espera de ellos y permite crear un itinerario por el que pueda transitar el alumnado en su escolarización. Así, un alumno o alumna puede completar ese camino, consumando paquetes formativos cada curso de escolaridad, que les reconozcan en sus expedientes académicos como activistas destacados en convivencia en positivo entre sus iguales.

\section{REFERENCIAS BIBLIOGRÁFICAS}

Alonso, MN N. y Avilés, J. M (2017a). Caderno de formação das equipes de ajuda. Alunos e alunas: Ensino Fundamental I. Americana, SP: Adonis.

Alonso, Ma N. y Avilés, J. Mª (2017b). Caderno de formação das equipes de ajuda. Professores e professoras: Ensino Fundamental I. Americana, SP: Adonis.

Avilés, J. Ma (2006). Bullying: el maltrato entre iguales. Agresores, víctimas y testigos en la escuela. Salamanca: Amarú. 
Avilés, J. Ma (2012). Manual contra el bullying. Guía para el profesorado. Lima: Libro Amigo.

Avilés, J. Ma (2013, agosto). Educação para a convivência. Ponencia presentada en el Seminário Internacional 'A convivência na escola em pauta', Campinas, SP. Brasil.

Avilés, J. Ma (2014, enero). Redes Sociales y Ciberconvivencia. Ponencia presentada en la I Jornada Provincial de Convivencia en los Centros, Valladolid.

Avilés, J. Ma (2015). Proyecto Antibullying. Madrid: CEPE.

Avilés, J. Ma (2018, en prensa). Los Sistemas de Apoyo entre Iguales en la escuela. Americana, SP: Adonis.

Avilés, J. Ma y Alonso, Mª N. (2017a). Caderno de formação das equipes de ajuda. Alunos e alunas: Ensino Fundamental II. Americana, SP: Adonis.

Avilés, J. Ma y Alonso, M N N. (2017b). Caderno de formação das equipes de ajuda. Professores e professoras: Ensino Fundamental II. Americana, SP: Adonis.

Avilés, J. Ma y García Barreiro, J. (2016, julio). Cibermentores. En línea: [http://convivenciaenlaescuela.es/, extraído el 16 de octubre de 2016]

Avilés, J. Ma , García, N. y Mateu, F. (2012). Tutores/as de convivencia, nuevos perfiles profesionales y educativos para la resolución de los conflictos en la escuela. Comunicación presentada en el $9^{\circ}$ Congreso de Psicología da Saúde, En J. L. Pais, I. Leal, A. Pereira, A. Torres, I. Direito y P. Vagos (Eds.), pp. 770-777 Aveiro: Placebo.

Avilés, J. Ma , Torres, N. y Vián, M.V. (2008). Equipos de ayuda, maltrato entre iguales y convivencia escolar. Electronic Journal of Research in Educational Psychology, 6, 3, 357-376.

Boqué, C. (2005). Tiempo de mediación. Sevilla: Consejería de Educación de la Junta de Andalucía.

Cerezo, F. (2001). La violencia en las aulas. Madrid: Pirámide.

Cohen, J.; Compton, R. y Diekmann, C. (2000). Conflict resolution education and social emotional learning programs: a critical comparison of school-based efforts. The Fourth R, 90, 1-6.

Close, C. L. y Lechman, K. (1997). Fostering youth leadership: students train students and adults in conflict resolution. Theory into Practice, 36, 11-17. DOI: https://doi. org/10.1080/00405849709543739

Cowie, H. y Fernández, F.J. (2006).Ayuda entre iguales en las escuelas: desarrollo y retos. Revista Electrónica de Investigación Psicoeducativa, 4 (2), 291-310

Cowie, H. y Jennifer, D. (2007). Managing violence in schools. A whole-school approach to best practice. London: PCP.

Cowie H y Oztug O. (2008). Pupils' perceptions of safety at school. Pastoral Care in Education, 26, (2), 59-67. DOI: https://doi.org/10.1080/02643940802062501

Cowie, H. y Sharp, S. (1996). Peer counseling schools. London: David Fulton Publishers.

Cowie, H. y Smith, P.K. (2010). Peer support as a means of improving school safety and reducing bullying and violence. En B. Doll, W. Pfohl, y J. Yoon (Eds.), Handbook of Prevention Research. New York: Routledge, pp.177-193. DOI: http://dx.doi.org/10.4324/9780203866412. $\underline{\operatorname{ch} 9}$

De Vicente, J. y Fernández, I. (2014) Estructuras escolares al servicio de la participación del alumnado. En CONVIVES, 7 (3), 22-31. En línea: [http://convivenciaenlaescuela.es/, extraído el 16 de enero de 2015].

Duran, D. y Vidal, V. (2004). Tutoría entre iguales: de la teoría a la práctica. Barcelona: Graó. 
Fernández, I. (2007). El estilo docente como fuente y resolución de conflictos. En J. Ma Avilés (Coord.) Convivencia Escolar, pp. 149-174.

Fernández, I. Villoslada, E. y Funes, S. (2002). Conflicto en el centro escolar. Madrid: Catarata.

García Barreiro, J. y Avilés, J. Ma (2018, en prensa). Sistemas de Mediación. En J. Ma Avilés (Coord.) Los Sistemas de Apoyo entre Iguales en la escuela. Americana: Adonis.

Gartner, A., Kohler, M.C. y Riessman, F. (1971). Children teach children. Nueva York: Harper Row. Hung, A. H., Luebbe, A. M. y Flaspohler, P. D. (2014). Measuring School climate: Factor analysis and relations to emotional problems, conduct problems, and victimization in Middle School Students. School Mental Health, 1-15.

Jones, T. (1998).Comprehensive Peer Mediation Evaluation Project. The fourth R, 82, 6-10.

Lester, L. y Cross, D. (2015). The Relationship Between School Climate and Mental and Emotional Wellbeing Over the Transition from Primary to Secondary School. Psychology of Well-Being, 5(1), 9. DOI: https://doi.org/10.1186/s13612-015-0037-8

LOE (2006). Ley Orgánica 2/2006, de 3 de mayo, de Educación. Boletín Oficial del Estado, nº 106, de 4 de mayo de 2006.

Luengo, J.A. (2014). Ciberbullying. Prevenir y actuar. Madrid: COP de Madrid. DOI: https://doi. org/10.5093/g201401

Megginson, D. y Clutterbuck, D. (2009). Further techniques for coaching and mentoring. London: Butterworth-Heinemann

Naylor, P. (2010). Anti-bullying peer support systems in school: What are they and do they work? Ponencia impartida en el II International Congress of Community Life at School. Almeria: Universidad de Almeria.

Naylor, P. y Cowie, H. (1999) The effectiveness of peer support systems in challenging school bullying: the perspectives and experiences of teachers and pupils, Journal of Adolescence, 22, 467-479. DOI: https://doi.org/10.1006/jado.1999.0241

Naylor, P. y Cowie, H. (2000). The role of peer support systems in education for citizenship, The School Field, 11, (1-2), 131-142.

Pellegrini, A. y Bartini, M. (2000). A longitudinal study of bullying, victimization, and peer affiliation during the transition from primary school to middle school. American Educational Research Journal, 37, (3), 699-725. DOI: https://doi.org/10.3102/00028312037003699

Rigby, K. (2002). New Perspectives on Bullying. Philadelphia: JKP.

Slavin, R.E. (1995). Cooperative learning. Theory, research and practice. Massachusetts: Allyn y Bacon.

Sullivan, K. (2001). The anti-bullying handbook. Singapore: Oxford University Press.

Tognetta, L., Avilés, J. M., Rosario, P. y Alonso, N. (2015). Moral disengagement, self-efficacy and bullying. Revista de Estudios e Investigación en Psicología y Educación, 2, (1), 30-34, DOI: https://doi.org/10.17979/reipe.2015.2.1.714

Topping, K. J. (1996). The effectiveness of peer tutoring in further and higher education: A typology and review of the literature. Higher Education, 32, 321-345. DOI: https://doi.org/10.1007/ $\underline{\mathrm{BF} 00138870}$

Torrego, J. C. (Ed.) (2001). Mediación de conflictos en instituciones educativas. Manual para la formación de mediadores. Narcea: Madrid. 
Vaello, J. (2003). Resolución de conflictos en el aula. Madrid: Santillana.

Van Slyck, M. y Stern, M. (1999). A developmental approach to the use of conflict resolution interventions with adolescents. En L. R. Forcey y I. M. Harris (eds.), Peace building for adolescents. Strategies for educators and community leaders (pp. 177-194). New York: Peter Lang.

Zaitegi, N., Fernández, I., Uruñuela, P., Avilés, J. Mª., Boqué, M. C., y Gómez, F. (2010). La educación en y para la convivencia positiva en España. Revista iberoamericana sobre calidad, eficacia y cambio en la educación, 8, (2), 93-132.

Zullig, K. J., Huebner, E. S. y Patton, J. M. (2011). Relationships among school climate domains and school satisfaction. Psychology in the Schools. 48 (2), 133-145. DOI: https://doi.org/10.1002/ pits. 20532 